\title{
Metastatic Breast Cancer Presenting as Refractory Anemia
}

By Julie Beaudoin-Maitre, MD and Chantal Vallée, MD

\begin{abstract}
About the Authors
Julie Beaudoin-Maitre is PGY 5 in the Department of Internal Medicine at the University of Sherbrooke, Sherbrooke, QC.

Chantal Vallée is an Assistant Professor, Department of Internal Medicine at the University of Sherbrooke, Sherbrooke, QC and Chief of Department of Specialized Medicine, CISSS de la Montérégie-Centre.
\end{abstract}

Correspondence to: julie.beaudoin-maitre@usherbrooke.ca

Submitted: December 20, 2017. Accepted: June 6, 2018. Published: February X, 2019. DOI: 10.22374/cjgim.v14i1.261

\begin{abstract}
We will review the case of a 71-year-old woman with an atypical presentation of stage IV breast cancer. She presented with symptomatic normocytic anemia ( $\mathrm{Hb} 85$ to $100 \mathrm{~g} / \mathrm{L}$ ) for the past two years, with a normal extended laboratory evaluation. Due to deterioration of her anemia, a bone marrow biopsy was done and concluded to myelodysplastic syndrome. Treatment with epoetin alfa and a trial of lenalidomide didn't improve her hemoglobin and she was still transfusion dependent. A second bone marrow biopsy revealed breast carcinoma infiltration, which was initially missed on the first biopsy. The patient was started on letrozole and palbociclib with good clinical response. Here, we present the clinical evolution, diagnosis and management of bone marrow micrometastasis due to breast cancer.

\section{Résumé}

Nous présentons l'étude de cas d'un cancer du sein de stade IV, chez une femme de 71 ans, ayant eu une présentation atypique. Elle s'est présentée avec une anémie normocytaire ( $\mathrm{Hb} 85$ à $100 \mathrm{~g} / \mathrm{L}$ ) symptomatique depuis deux ans, non explicable par un bilan biochimique approfondi. Suite à la dégradation de son anémie, une biopsie de moelle osseuse a été effectuée concluant à un syndrome myélodysplasique. Un traitement à l'epoetin alfa ainsi qu'un essai de lenalidomide ne permirent pas l'amélioration de son hémoglobine et elle est demeurée dépendante aux transfusions. Une seconde biopsie de moelle osseuse démontra une infiltration par un carcinome mammaire qui navait pas été constatée lors de la première biopsie. Un traitement avec du letrozole et du palbociclib a alors été débuté avec une réponse clinique satisfaisante. Nous passons en revue la présentation clinique, la démarche diagnostique ainsi que la prise en charge des micro métastases de la moelle osseuse dans le cancer du sein.
\end{abstract}

Keywords: metastatic breast cancer, bone marrow micrometastasis, cytopenia. 
Breast cancer is the most common cancer affecting women in Canada, with an estimated 25,700 new cases each year. It is the second most prevalent cause of cancer death in women, ${ }^{1}$ particularly because of distant metastases. Indeed, approximately $15 \%$ of women with operable breast cancer relapses within 15 years. ${ }^{2}$ In pooled multicenter analysis, bone marrow micrometastasis (BMM) was detected in $30 \%$ of patients with breast cancer stage I, II, and III. The presence of micrometastasis was an independent prognostic factor of poor outcome. ${ }^{3}$

While BMM is considered relatively frequent, the development of cytopenia and bone marrow failure is rare. ${ }^{4}$ Here we present a patient with progressive symptomatic anemia over three years, who was initially diagnosed with myelodysplastic syndrome but was later found to be a manifestation of breast cancer metastasis to the bone marrow.

\section{Case Presentation}

A 71-year old female patient was referred to our service for evaluation of a heterogeneous aspect of the thoracic spine on a chest computed tomography (CT). Her past medical history is significant for hypertension, hyperlipidemia, subarachnoid hemorrhage with a type I Chiari malformation, and a bioprosthetic aortic valve. The initial skeletal survey and bone scan were negative for metastasis. Blood tests revealed normocytic anemia, with a hemoglobin 85 to $100 \mathrm{~g} / \mathrm{L}$ and a mean corpuscular value of $95 \mathrm{fL}$ for the past two years. The white cells and platelets were within the range of normal. The extended laboratory evaluation for anemia was normal. Bone marrow evaluation was done in the following months, because of progressively worsening symptomatic anemia. The bone marrow aspiration was not diagnostic due to a dry tap, while the biopsy showed an hypercellular bone marrow with erythroid dysplasia, megakaryocytes and myelofibrosis grade 1 and 2, compatible with myelodysplastic syndrome. Patient was referred to a hematologist and started on epoetin alfa. Her hemoglobin improved with higher doses of epoetin, but red cell transfusions were still needed every four to six weeks to maintain a hemoglobin above $80 \mathrm{~g} / \mathrm{L}$. A trial of lenalidomide was stopped after one month, because there was no improvement of the anemia and the patient presented a skin rash. The patient later developed an iron overload due to the transfusions.

Since the anemia didn't respond as expected with typical treatment of myelodysplastic syndrome, a second bone marrow biopsy was done eight months after the first one and revealed breast carcinoma infiltration with positive estrogen receptor, negative HER2 and progesterone receptor. In light of the findings, the first biopsy was reviewed and showed positive epithelial cells to cytokeratin AE1/A3, GATA 3 and slightly positive to CD 138, consistent with a mammary carcinoma infiltration of $10-15 \%$ of the specimen surface. Those cells were probably mistaken on the first biopsy for granulocytic precursors cells. Based on these results, a review of the patient's gynecological history was taken. There was no past or familial history of breast or ovarian cancer. Her mammography's of the last two years were negative, the last one done a month prior. The physical exam was within normal and didn't reveal breast lump or lymphadenopathy. Tumour marker panel indicated increased serum level of Ca15-3, Ca19-9 and CEA. LDH were normal. The patient underwent a skeletal scintigraphy, which was remarkable for many bilateral costal lesions. An abdominal and pelvic CT scan confirmed the presence of diffuse lytic lesions and right axillary lymphadenopathy, which has been stable for the past two years. Diffuse metastasis of the spine was revealed on magnetic resonance imaging (MRI). No primary breast lesion was found on echography or MRI. After discussion, with the oncology team, we decided against doing a positron emission tomography (PET) scan since it wouldn't change the management of the cancer.

Patient was started on letrozole and palbociclib, an highly selective inhibitor of CDK 4/6. Cytopenia improved significantly in the next 6 months, with hemoglobin level of $112 \mathrm{~g} / \mathrm{L}$ and normalization of white blood cells and platelets level. A single dose of palliative radiotherapy was delivered on the left knee to alleviate the pain from metastasis infiltration.

\section{Discussion}

Patients presenting with chronic unexplained cytopenia can be a clinical challenge and warrant an extensive evaluation. The

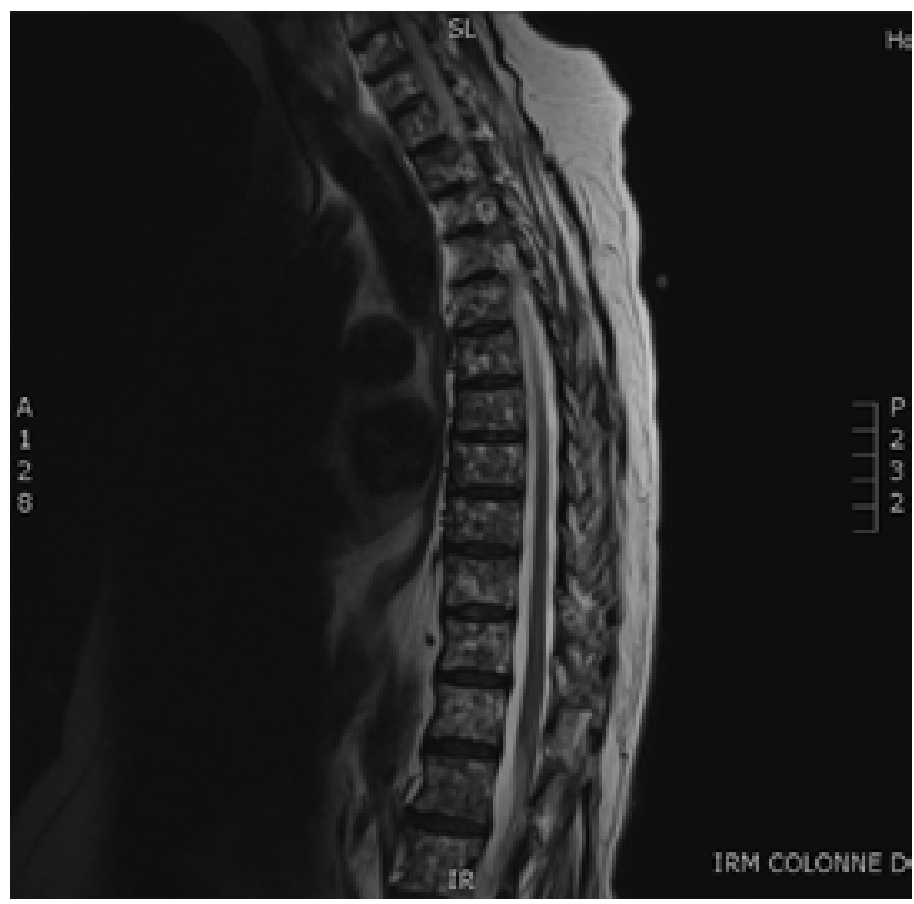

Figure 1. Diffuse metastatic infiltration of the spine on magnetic resonance imaging 
differential diagnosis is large and include nutritional deficiency, chronic inflammatory reactions, autoimmune diseases (e.g., autoimmune hemolytic anemia), chronic renal or hepatic diseases, infectious disorders, inherited conditions and bone marrow failure. Several causes of bone marrow failure need to be considered, such as myelodysplastic syndrome, aplastic anemia, infiltration of the bone marrow by hematologic or nonhematologic neoplasm, myelofibrosis or toxic damage. ${ }^{5}$ In our case, the second bone marrow biopsy was diagnostic for breast cancer bone marrow infiltration.

While breast cancer is commonly diagnosed in the early stages of the disease without evidence of distant metastases, recurrence at distant sites may arise years after the initial diagnosis and treatment. We now know that breast cancer has an early spread of tumour cells. ${ }^{6}$ The dissemination of tumour cells can take two pathways, either lymphatic dissemination or hematogenous dissemination. The detection of BMM is evidence of dissemination through the blood circulation. ${ }^{7}$

As reported earlier, a third of patients have BMM detected at the time of breast cancer diagnosis. ${ }^{3}$ The majority of those patients have normal blood counts and no specific symptoms. A previous study (Kopp et al.) reported an incidence of only $0.17 \%$ clinically apparent BMM. The actual incidence may be underestimated, as stated by the investigators, because the diagnosis may have been missed and their databased was incomplete. ${ }^{4}$

When BMM is clinically evident, the most common finding is anemia, present in $40-60 \%$ of patients, while $12-25 \%$ of patients have leukopenia and thrombocytopenia. ${ }^{8}$ Other laboratory abnormalities include hypoproteinemia and elevated serum lactate dehydrogenase. ${ }^{9}$ Symptoms reported are asthenia, anorexia and bone pain secondary to osteolytic lesions. In a past series of 22 cases, a close association between bone metastasis and bone marrow involvement was confirmed, with all patients having bone metastasis ${ }^{4}$. Other studies found that $2-8 \%$ of patients with BMM had no evidence of skeletal involvement. ${ }^{9}$

Demir et al, reported that the median time to diagnosis of BMM was of 3 years after the initial diagnosis of breast cancer. The interval was shorter in hormonal receptor negative tumoUrs $(17.9 \text { months })^{10}$ which is consistent with past studies. Risk factors for BMM include large tumoUr size, poor differentiation, lymph node metastasis, and negative hormone receptors. ${ }^{3}$ There are some reports of bone marrow involvement as the initial presentation of breast cancer. ${ }^{11}$ To our knowledge, there is no other reported case of evidence of BMM months before the diagnosis of breast cancer without breast lesion on physical exam and a normal mammography.

Non-invasive test for diagnosis of BMM include wholebody PET, with a sensitivity of $87 \%$ with a positive predictive value of $94 \%$. Since most patients undergo PET scan for the stating of the disease, PET seems the modality of choice for the diagnosis of BMM. Whole-body PET with 18F-FDG exploits the high glycolytic rate of malignant tissue compared with that of non-malignant cells, which can reveal previously unknow metastatic disease to the bone marrow. ${ }^{12}$ Peripheral blood smear can show signs of marrow infiltration with the presence of leukoerythroblastosis, if other causes, such as myelodysplastic syndrome and myeloproliferative syndromes are ruled out. Leukoerythroblastosis is defined as the presence of nucleated erythrocytes and immature myeloid cells in the peripheral blood. ${ }^{13}$ Bone marrow evaluation is often needed to confirm the diagnosis. Past series suggested that bone marrow biopsy is more useful than aspiration for the diagnosis of BMM, ${ }^{14}$ with less than $15 \%$ of false-negative results. ${ }^{8}$

There is limited literature regarding the safest and most effective treatment of patients with BMM. The presence of cytopenia, due to breast cancer infiltration of the marrow, poses a difficult problem in the treatment of affected patients. Past reports demonstrated the need for intensive hematological support in more than 50\% of patients treated with full-dose chemotherapy and a risk of infection around 20\%. ${ }^{14-16}$ More recently, small studies have presented benefit, including improvement of cytopenia, with the use of low-dose chemotherapy with capecitabine, endocrine therapy, anthracycline or trastuzumab. ${ }^{8-18}$ More studies are needed before a standard regimen can be established.

The prognosis of breast cancer patients with BMM, while variable, is usually poor. The median survival time after the diagnosis of apparent BMM varied from more than 6 to 19 months, in past studies. ${ }^{4-10}$ The extent of bone marrow infiltration may have prognostic value. In a multivariate analysis of prognostic factors in patients with breast cancer, a pre-treatment hemoglobin less than $110 \mathrm{~g} / \mathrm{L}$ and platelets below $100 \times 10^{9} / \mathrm{L}$ was associated with a poor prognostic. ${ }^{8-19}$ BMM may be an indicator of early recurrence. Cote et al, reported an association between the presence of micrometastasis in operable breast cancer stage I and II, and early recurrence. The 2 years' recurrence rates were $3 \%$ and $33 \%$ in 49 patients without and with micrometastases respectively. ${ }^{20}$

\section{Conclusion}

In conclusion, unexplained cytopenia is a strong indicator of the necessity of bone marrow examination. Bone marrow is a frequent site of metastasis of breast cancer and other solid tumours. Patients with BMM usually don't have bone marrow failure, but they can present with anemia, thrombocytopenia or pancytopenia. Further studies are needed to develop more effective therapies. 


\section{References}

1. Canadian Cancer Society's Advisory Committee on Cancer Statistics. Canadian Cancer Statistics 2016. Toronto, ON: Canadian Cancer Society; 2016.

2. Obedian E, Fischer DB, Haffty BG. Second malignancies after treatment of early-stage breast cancer: lumpectomy and radiation therapy versus mastectomy. J Clin Oncol 2000;18:2406-12.

3. Braun S, Vogl FD, Naume B, Janni W, et al. A pooled analysis of bone marrow micrometastasis in breast cancer. N Engl J Med 2005;353:793-802.

4. Kopp, Hans-Georg, et al. Symptomatic bone marrow involvement in breast cancer-clinical presentation, treatment, and prognosis: a single institution review of 22 cases. Anticancer Res 2011;31.11:4025-30.

5. Valent P. Low blood counts: immune mediated, idiopathic, or myelodysplasia, Am Soc Hematol 2012;485-91.

6. Klein CA. Parallel progression of primary tumours and metastases. Nat Rev Cancer 2009;9(4):302-12.

7. Hartkopf AD, Banys M, Krawczyk N et al. Bone marrow versus sentinel lymph node involvement in breast cancer: a comparison of early hematogenous and early lymphatic tumor spread. Breast Cancer Res Treat 2012;131:501-508.

8. Freyer G, Blandine L, and Trillet-Lenoir V. Palliative hormone therapy, lowdose chemotherapy, and bisphosphonate in breast cancer patients with bone marrow involvement and pancytopenia: report of a pilot experience. Eur J Intern Med 2000;11(6):329-33.

9. Ozkalemkas F, Ali R, Ozkocama V et Al. The bone marrow aspirate and biopsy in the diagnosis of unsuspected nonhematologic malignancy: A clinical study of 19 Cases. BMC Cancer 2005;5:144.
10. Demir L, Akyol M, Bener, S et al. Prognostic evaluation of breast cancer patients with evident bone marrow metastasis. Breast J 2014;20:279-87.

11. Duma N, Wang Z, Miranda C. Bone Marrow involvement as the initial presentation of breast cancer. Am J Med Case Rep 2015;3(6):177-80.

12. Lewanski, Conrad R., et al. Bone marrow involvement in breast cancer detected by positron emission tomography. J Royal Soc Med 1999;92(4):193.

13. Shamdas GJ, Ahmann FR, Matzner MB and Ritchie JM: Leukoerythroblastic anemia in metastatic prostate cancer. Clinical and prognostic significance in patients with hormone refractory disease. Cancer 1993;71:3594-600.

14. Contreras E, Ellis LD, Lee RE. Value of bone marrow biopsy in the diagnosis of metastatic carcinoma. Cancer 1972;29:778-84.

15. Rodriguez-Kraul R, Hortobagyi GN, Buzdar AU et al. Combination chemotherapy for breast cancer metastatic to bone marrow. Cancer 1981;48:227-38.

16. Ingle JN, Tormey DC, Bull JM et al. Bone marrow involvement in breast cancer: effect on response and tolerance to combination chemotherapy. Cancer 1977;39:250-7.

17. Ardavanis A, Kountourakis P, Orphanos G and Rigatos G. Low-dose capecitabine in breast cancer patients with symptomatic bone marrow infiltration: a case study. Anticancer Res 2008;28:539-42.

18. Xu L, Guo F, Song S et al. Trastuzumab monotherapy for bone marrow metastasis of breast cancer: A case report. Oncol Lett 2014;7:1951-53.

19. Hortobagyi GN, Smith TL, Legha SS et al. Multivariate analysis of prognostic factors in advanced breast cancer. J Clin Oncol 1983;1:1776-82.

20. Cote RJ, Rosen PP, Lesser ML, Old LJ, Osborne MP. Prediction of early relapse in patients with operable breast cancer by detection of occult bone marrow micrometastases. J Clin Oncol 1991;9:1749-56. 